\title{
Adaptive Platoon Control for Marine Surface Vehicles in the Presence of Constraints
}

\author{
Xiaoling Liang $\mathbb{D}^{1}$ and Duansong Wang $\mathbb{D}^{2}$ \\ ${ }^{1}$ Department of Marine Engineering, Dalian Maritime University, Dalian 116026, China \\ ${ }^{2}$ College of Automation, Harbin Engineering University, Harbin 150001, China \\ Correspondence should be addressed to Xiaoling Liang; lxl_dmu2008@126.com
}

Received 21 April 2020; Revised 4 July 2020; Accepted 11 July 2020; Published 5 August 2020

Academic Editor: Xianming Zhang

Copyright (c) 2020 Xiaoling Liang and Duansong Wang. This is an open access article distributed under the Creative Commons Attribution License, which permits unrestricted use, distribution, and reproduction in any medium, provided the original work is properly cited.

\begin{abstract}
This paper presents the platoon formation control design for unmanned surface vehicles with input and output constraints. First, Barrier Lyapunov Function (BLF) is employed to ensure the desired line-of-sight (LOS) range. The LOS range should be maintained within the predefined regions between each marine vehicle and its predecessor while the connected platoons track the trajectory. Next, to handle the model uncertainties and unknown external disturbance, we propose an adaptive neural network controller that approximates the unknown nonlinearities. Furthermore, the Nussbaum function is applied to compensate for input saturations. In addition, formation errors can be guaranteed by stability analysis. The distance between two consecutive agents does not violate collision avoidance and connectivity in the presence of input saturation. Finally, the feasibility of the theoretical results is illustrated through the simulation results.
\end{abstract}

\section{Introduction}

Multiagent systems have been an active area due to higher efficiency, stronger robustness, and less communication requirement [1-3]. Recently, many effective schemes have been developed to solve the formation control problem, such as sliding mode control [4-6] and backstepping control [7-11]. Platoon control strategy has played an increasingly important role in decentralized formation fields [12-14], which brings many benefits such as less energy consumption and more channel capacity. The robust $H_{\infty}$ control method was proposed to design platoon velocity profile which promotes smoothness of traffic flow [15]. Traffic efficiency was studied with the help of vehicular networking to optimize traffic flow [16]. Existing works mostly focus platoon formation on the development of the automobile industry. However, extensive applications on marine control are seldom considered. Inland navigation and channel transshipment are important components of waterborne transport. Marine vessel train consists of a lead vessel followed by a series of unmanned follower vessels. Motivated by the previous discussion, this paper investigates the issue of adaptive platoon formation control of marine surface vehicles.

Dynamic disturbances and unmodeled dynamics widely exist in many physical systems, such as power industries $[17,18]$ and aerospace systems $[19,20]$. Inherently, nonlinear and uncertainty characteristics of system dynamics are difficult to model. In addition, ignoring the unknown disturbances in controller design, the precision and robustness will have an effect on the performance of the closed-loop system. Neural network has the ability to adjust the parameters adaptively [21]. Comparing with the existing work, modelbased control [22] and the linearity-in-parameter assumption $[23,24]$ are not required. Due to extreme or varying environments, the external forces such as wind, wave, and current are involved into vessel motion. Many academicians and engineers have dedicated to adaptive control of marine surface vehicles. To overcome the nonlinear uncertainty and external disturbance, adaptive neural control schemes are proposed for multiple marine surface vehicles with model uncertainties in platoon formation cases. 
Driven by practical needs and theoretical challenges, the constrained control design of practical systems has become an interesting research topic [25-27]. In practical systems, input saturation and output constraint cause performance degradation or even instability [28]. How to achieve and maintain a desired formation geometry in these situations is one of the fundamental issues in platoon. If the relative motion states are constrained within the predefined regions, two consecutive vehicles can avoid undesired collision in the procedure of platoon phase. This problem is generally described by line-of-sight (LOS) range and angle as output constraint. To ensure output constraint, barrier Lyapunov function (BLF) is a remarkable concept to deal with output constraints. Various schemes based on BLF have been reported for marine vessels [29-31]. On the basis of a previous successful work, BLF in the current study is further applied to MSVs by solving constraints of LOS range and angle. Furthermore, the requirements of limited input amplitude should be incorporated into the platoon formation design. Actuator saturation is another important factor to a practical system. Many research results have been carried out on input saturation. In [32], adaptive fuzzy tracking was developed for uncertain multi-input multi-output (MIMO) nonlinear systems in the presence of input saturation. In [33], the auxiliary system based on backstepping design is employed to cope with input constraints. No assumption on the uncertain parameters is needed within the compact set. Decentralized neural network based on adaptive backstepping scheme was presented in [34] to deal with time delay and input nonsmooth constraint. However, in all the papers mentioned above, there is little information on how to handle the two challenging constraints for the platoon formation. Therefore, it is meaningful to consider the platoon formation subject to output constraint and input saturation.

Marine vessels have characteristics of coupled dynamics, nonlinearities, and parametric uncertainties. Actually, the requirements of safety, reliability, robustness, and limited input amplitude should be improved in multiple marine vessels. Motivated by the aforementioned discussion, a platoon formation control approach of multiple marine vessels has been developed to simultaneously deal with output constraint and input saturation. BLF in adaptive backstepping design is proposed to ensure the platoon in the safe ranges. The unknown external disturbance and unmodeled dynamics are approximated by using neural network. The problem of multiple marine vehicles with actuator saturations is also considered in this paper. The main contributions of the proposed schemes are highlighted as follows:

(i) Output constraints on marine vessels positioning within line-of-sight cone is proceeded under the framework of adaptive backstepping design. LOS range and angle errors in formation operations of unmanned surface vehicles are handled by barrier Lyapunov function.

(ii) Adaptive backstepping is applied to parametric-uncertain systems. Unknown disturbances and parametric uncertainties are compensated by adaptive neural network control. Backstepping control provides the systematic and recursive design.

(iii) An auxiliary system is employed to cope with physical constraints of the actuators. In order to ensure the constraints on thrust magnitude, Nussbaum function is introduced to facilitate the closed-loop system design. Compared with other algorithms dealing with input constraints, this approach avoids the discontinuous switching mechanism [32] and singularity in [35].

The rest of the paper is divided into four sections. Section 2 describes the problem formation for platoon formation and preliminaries. Section 3 presents the controller design process, and the stability of the multiple marine vessels is rigorously analyzed. In Section 4, numerical simulations are conducted to support the theoretical developments. Finally, conclusions are drawn in Section 5.

\section{Preliminaries and Problem Description}

2.1. Problem Description. We consider $n+1$ marine surface vehicles labeled as 0 to $n$. The formation pattern of this class multiagent system is shown in Figure 1. The kinematics and dynamics of the $i$-th MSV can be modeled as follows:

$$
\begin{aligned}
\dot{\eta}_{i} & =J_{i}\left(\eta_{i}\right) v_{i}, \\
M_{i} \dot{v}_{i} & =-C_{i}\left(v_{i}\right) v_{i}-D_{i}\left(v_{i}\right) v_{i}+\bar{d}_{i}+\Delta_{i}+\operatorname{sat}\left(\tau_{i}\right),
\end{aligned}
$$

where $\eta_{i}=\left[x_{i}, y_{i}, \psi_{i}\right]^{T}$ denotes the MSV position and yaw angle with respect to earth-fixed frame, $v_{i}=\left[u_{i}, v_{i}, r_{i}\right]^{T}$ represents the orientation known as surge, sway, and yaw velocities in the body-fixed frame, $\bar{d}_{i}$ is external disturbances induced by wind, wave, and ocean currents, $\Delta_{i}$ represents the unmodeled dynamics, $\tau_{i}$ denotes the actual control inputs of the $i$-th $\mathrm{MSV}, J_{i}\left(\psi_{i}\right)$ is a nonsingular transformation matrix, $M_{i}$ is the inertia matrix, $C_{i}$ is the skew-symmetric matrix of Coriolis and centripetal terms, and $D_{i}$ is the damping matrix.

From a practical point of view, the control forces and moments of the marine vessels are limited by the physical properties of thrusters. The saturation nonlinearities can be described as

$$
\operatorname{sat}\left(\tau_{i}\right)= \begin{cases}\operatorname{sign}\left(\tau_{i}\right) \tau_{i \max }, & \text { if }\left\|\tau_{i}\right\|>\tau_{i \max }, \\ \tau_{i}, & \text { if }\left\|\tau_{i}\right\|<\tau_{i \max },\end{cases}
$$

where $\tau_{\text {imax }}$ stands for the maximum control forces or moments of vessels' propulsion systems:

$$
g_{i}\left(\tau_{i}\right)=\tau_{i \max } \times \tanh \left(\frac{\tau_{i}}{\tau_{i \max }}\right)=\tau_{i \max } \frac{e^{\left(\tau_{i} / \tau_{i \max }\right)}-e^{\left(-\tau_{i} / \tau_{\max }\right)}}{e^{\left(\tau_{i} / \tau_{i \max }\right)}+e^{\left(\tau_{i} / \tau_{i \max }\right)}} .
$$

Then, we obtain

$$
\operatorname{sat}\left(\tau_{i}\right)=g_{i}\left(\tau_{i}\right)+d_{1 i}
$$

The relationship between the saturation and approximation function is shown in Figure 2. Then, the augmented system considering the saturation approximation function is 


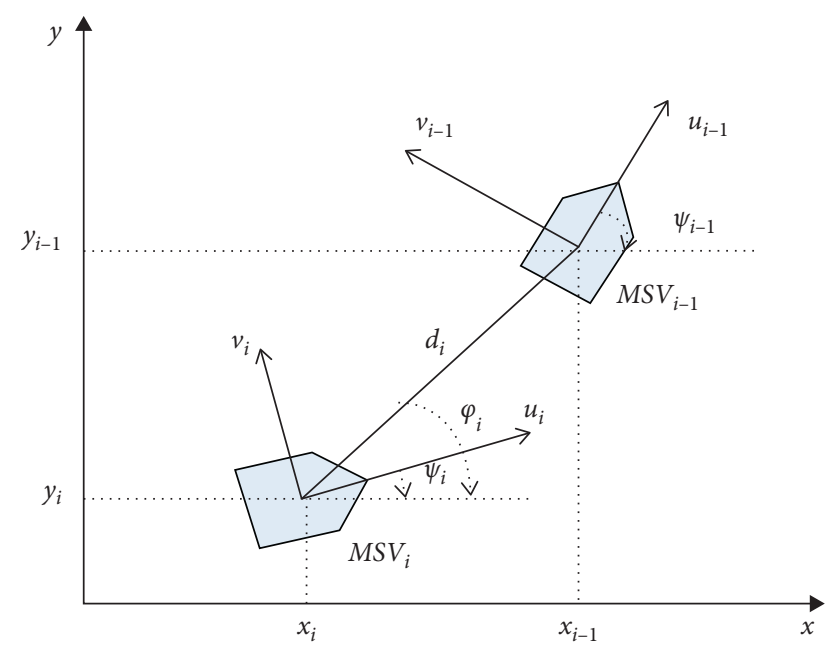

FIGURE 1: MSVs formation configuration.

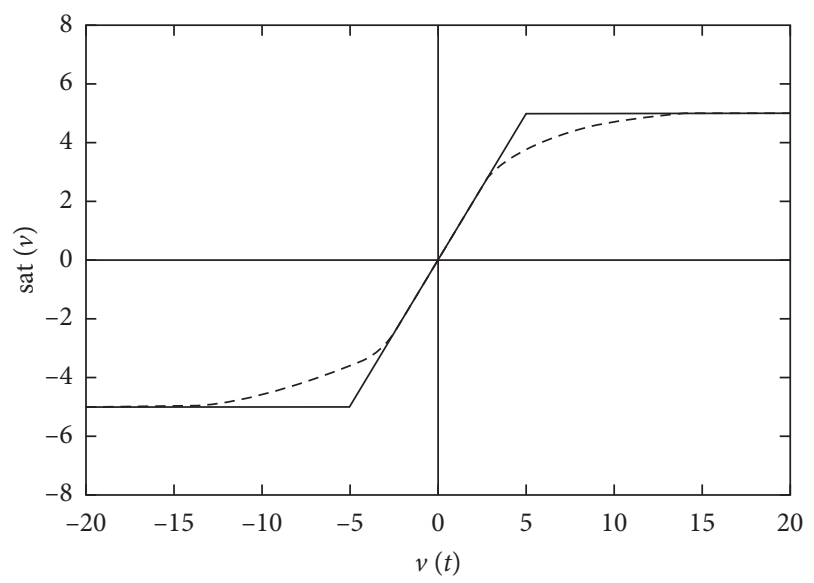

FIgURE 2: Saturation (dotted line: approximate function; solid line: $\operatorname{sat}(\mathrm{v}))$.

$$
\begin{aligned}
M_{i} \dot{v}_{i} & =-C_{i}\left(v_{i}\right) v_{i}-D_{i}\left(v_{i}\right) v_{i}+d_{i}+\Delta_{i}+g_{i}\left(\tau_{i}\right), \\
T_{i} \dot{\tau}_{i}+\tau_{i} & =\tau_{c i},
\end{aligned}
$$

where $\tau_{c i}$ describes the control command signal that we should design, $T_{i} \in \mathbb{R}^{3 \times 3}$ is a diagonal matrix of thruster time constant, and the 'disturbance-like' term is denoted as

$$
d_{i}=d_{1 i}+\bar{d}_{i}
$$

Assumption 1. The desired reference trajectory $\eta_{0}=\left[x_{0}, y_{0}, \psi_{0}\right]^{T}$ and its first time derivative $\dot{\eta}_{0}$ are bounded functions.

Assumption 2. The external disturbances $\bar{d}_{i}$ are bounded with unknown constants. Then, according to equation (7), there exist positive constants $D_{i \max }$ such that $d_{i} \leq D_{i \max }$.

In this section, the platoon formation problem is considered for MSVs modeled by (1) and (2) with uncertainties and constraints. We will design a control strategy for the control objectives as follows:

(1) A desired line-of-sight range between two consecutive MSVs can be achieved under collision and connectivity constraints.

(2) Modelling uncertainties and external disturbances should be compensated, which can improve the system performance.

(3) A string of MSVs proceed along a given trajectory in a platoon configuration. All MSVs can maintain the desired position in a decentralized formation.

\subsection{Preliminaries}

Lemma 1 (see [31]). For any constant $x \in \mathbb{R}^{n}$, if $|x|<k$ and $k$ is a constant, then the following inequality holds

$$
\ln \frac{k^{2}}{k^{2}-x^{2}} \leq \frac{x^{2}}{k^{2}-x^{2}} \text {. }
$$

Lemma 2 (see [36]). Let $N(\chi)$ be an even Nussbaum function and $V(\cdot)$ and $\chi_{i}(\cdot)$ be smooth functions defined on $\left[0, t_{f}\right)$, with $V(t) \geq 0, \forall t \in\left[0, t_{f}\right)$. If the following inequality holds,

$$
V \leq V(0) e^{-c t}+\frac{M}{c}\left(1-e^{-c t}\right)+\frac{e^{-c t}}{r_{\chi}} \int_{0}^{t} \sum_{i=1}^{n}\left(\varepsilon_{i} N\left(\chi_{i}\right) \dot{\chi}_{i}-\dot{\chi}_{i}\right) e^{c \tau} \mathrm{d} \tau,
$$

where $c>0, r_{\chi}>0, \varepsilon_{\dot{p}_{t}}>0$, and $M$ is a positive constant, then $V(\cdot)$ and $\chi_{i}(\cdot)$ and $\int_{0}^{t} \sum_{i=1}^{n}\left(\varepsilon_{i} N\left(\chi_{i}\right) \dot{\chi}_{i}-\dot{\chi}_{i}\right) e^{c \tau} d \tau$ are bounded on $\left[0, t_{f}\right)$.

\section{Control Design with Barrier Lyapunov Function}

3.1. Formation Control of MSVs. In this section, we consider the communications among $n+1$ MSVs involving a LOS guidance system. First, a LOS guidance procedure is derived in this section. The desired output is reduced from $\left(x_{i}, y_{i}, \psi_{i}\right)$ to $\left(d_{i}, \psi_{i}\right)$ based on LOS projection algorithm. The LOS projection algorithm will guarantee that the task of tracking trajectory is satisfied. LOS geometric task is to force the vessel position $\left(x_{i}, y_{i}\right)$ to converge to a desired path by following its leader. Line-of-sight (LOS) range, $d_{i}$, and angle, $\varphi_{i}$, between two MSVs are defined as

$$
\begin{aligned}
& d_{i}=\sqrt{\left(x_{i-1}-x_{i}\right)^{2}+\left(y_{i-1}-y_{i}\right)^{2}}, \\
& \varphi_{i}=\arctan 2\left(y_{i-1}-y_{i}, x_{i-1}-x_{i}\right) .
\end{aligned}
$$

The formation errors of the MSVs are designed as

$$
\begin{aligned}
& e_{d i}=d_{i}-d_{i, \mathrm{des}}, \\
& e_{\psi i}=\psi_{i-1}-\psi_{i},
\end{aligned}
$$

where $d_{i, \text { des }}$ is a desired distance between two MSVs. According to equation (11), differentiating $e_{d i}$ and $e_{\psi i}$ with respect to time, we can obtain 


$$
\begin{aligned}
\dot{e}_{d i}= & -\left(z_{21 i}+\alpha_{1 i}\right) \cos \left(\psi_{i}-\varphi_{i}\right)+\dot{y}_{i-1} \sin \varphi_{i}+\left(z_{22 i}+\alpha_{2 i}\right) \sin \\
& \cdot\left(\psi_{i}-\varphi_{i}\right)+\dot{x}_{i-1} \cos \varphi_{i}, \\
\dot{e}_{\psi i}= & \dot{\psi}_{i-1}-\left(z_{23 i}+\alpha_{3 i}\right) .
\end{aligned}
$$

In order to avoid collision and connectivity maintenance among vehicles, the desired distance during the whole moving process must satisfy the following equations:

$$
0<d_{i, \operatorname{min~col}}<d_{i} \leq d_{i, \max c o m},
$$

where $d_{i, \min \text { col }}$ and $d_{i, \max \text { com }}$ represent the minimum safety distance and maximum effective communication distance respectively. For convenience, we define the maximum and minimum error distance as

$$
\begin{aligned}
& \underline{e}_{d i}(t)=d_{i, \text { min col }}-d_{i, \text { des }}, \\
& \bar{e}_{d i}(t)=d_{i, \text { max com }}-d_{i, \text { des }} .
\end{aligned}
$$

The bounds of yaw angle errors are defined as $\bar{e}_{\psi i}$ and $\underline{e}_{\psi i}$. Kinematic constraints are generally considered from the motion planning. Based on coordinate transformation, kinematic constraints have been transferred to collision avoidance and connectivity maintenance constraints based on LOS range and angle. The constraints of the errors are given as follows:

$$
\begin{aligned}
& \underline{e}_{d i}<e_{d i}<\bar{e}_{d i}, \\
& \underline{e}_{d i}<e_{\psi i}<\bar{e}_{\psi i} .
\end{aligned}
$$

Define the tracking errors as

$$
\begin{aligned}
& z_{1 i}=\left[z_{11 i}, z_{12 i}\right]^{T}=\left[e_{d i}, e_{\psi i}\right]^{T}, \\
& z_{2 i}=\left[z_{21 i}, z_{22 i}, z_{23 i}\right]^{T}=v_{i}-\alpha_{i},
\end{aligned}
$$

where $\alpha_{i}=\left[\alpha_{1 i}, \alpha_{2 i}, \alpha_{3 i}\right]^{T}$ is a stabilizing function designed as follows:

$$
\begin{aligned}
& \alpha_{1 i}=\cos \left(\psi_{i}-\varphi_{i}\right)\left[k_{d i} e_{d i}\left(k_{a i}^{2}-e_{d i}^{2}\right)+\dot{x}_{i-1} \cos \varphi_{i}+\dot{y}_{i-1} \sin \varphi_{i}\right], \\
& \alpha_{2 i}=-\sin \left(\psi_{i}-\varphi_{i}\right)\left[k_{d i} e_{d i}\left(k_{a i}^{2}-e_{d i}^{2}\right)+\dot{x}_{i-1} \cos \varphi_{i}+\dot{y}_{i-1} \sin \varphi_{i}\right], \\
& \alpha_{3 i}=k_{\psi i} e_{\psi i}+\dot{\psi}_{i-1} .
\end{aligned}
$$

Differentiating $z_{2 i}$ with respect to time, we can get

$$
M_{i} \dot{z}_{2 i}=-C_{i}\left(v_{i}\right) v_{i}-D_{i}\left(v_{i}\right) v_{i}+g_{i}\left(\tau_{i}\right)+d_{i}+\Delta_{i}-M_{i} \dot{\alpha}_{c i} \text {, }
$$

The continuous nonlinear function related to the speed $D_{i}\left(v_{i}\right) v_{i}$ and the unmodeled dynamics of the MSV are all unknown. To solve these problems, RBFNN is used to estimate the unknown dynamics and hydrodynamic damping terms. We give

$$
W_{i}^{* T} H_{i}\left(Z_{i}\right)+\varepsilon_{i}\left(Z_{i}\right)=-D_{i}\left(v_{i}\right) v_{i}+\Delta_{i} .
$$

Here, $Z_{i}=v_{i}$ is the inputs of the NN, $W_{i}^{*}$ is the true constant weight value, $H_{i}\left(Z_{i}\right)$ is the radial basis function, $\varepsilon_{i}\left(Z_{i}\right) \leq \bar{\varepsilon}_{i}$ is the approximate error, $\bar{\varepsilon}_{i}>0$ is an unknown arbitrary small constant, and $\widehat{W}_{i}^{T} H_{i}\left(Z_{i}\right)$ is used to approximate $W_{i}^{*} \mathrm{~T} H_{i}\left(Z_{i}\right)$. The adaptive update law is designed as follows:

$$
\dot{\hat{W}}_{i}=\Gamma_{i}\left(H_{i}\left(Z_{i}\right) z_{2 i}-\sigma_{i}\left|z_{2 i}\right| \widehat{W}_{i}\right), \quad i=1,2,3,
$$

where $\Gamma_{i}=\Gamma_{i}^{\mathrm{T}}>0$ are adaptive gain matrices and $\sigma_{i}$ is a positive constant.

The adaptive neural network control law is proposed as follows:

$$
\begin{aligned}
\alpha_{c i}= & \left(z_{2 i}^{T}\right)^{+}\left(-\frac{k_{d i} e_{d i}^{2}}{k_{a i}^{2}-e_{d i}^{2}}-\frac{k_{\psi i} e_{\psi i}^{2}}{k_{b i}^{2}-e_{\psi i}^{2}}\right)+C_{i}\left(v_{i}\right) v_{i}-\Theta_{2 i}+M_{i} \dot{\alpha}_{i} \\
& -K_{2 i} z_{2 i}-\widehat{W}_{i}^{\mathrm{T}} \mathrm{H}_{i}\left(Z_{i}\right),
\end{aligned}
$$

where $(*)^{+}$is the Moore-Penrose pseudoinverse of $(*)$ and the control gain matrix $K_{2 i} \in \mathbb{R}^{3 \times 3}>0$. Denote the 3 rd tracking error as

$$
z_{3 i}=g_{i}\left(\tau_{i}\right)-\alpha_{c i} .
$$

The saturation of the thrusters has been considered in the abovementioned equation. Then, we have

$$
\dot{z}_{3 i}=\xi_{i}\left(-\frac{1}{T_{i}} \tau_{i}+\frac{1}{T_{i}} \tau_{c i}\right)-\dot{\alpha}_{c i},
$$

where $\xi_{i}=\operatorname{diag}\left(\xi_{i 1}, \xi_{i 2}, \xi_{i 3}\right) \quad$ and $\quad \xi_{i j}=\partial g_{i j}\left(\tau_{i j}\right) / \partial \tau_{i j}$, $j=1,2,3$. We introduce Nussbaum function matrix $N_{i}=\operatorname{diag}\left[N_{i 1}\left(\chi_{i 1}\right), N_{i 2}\left(\chi_{i 2}\right), N_{i 3}\left(\chi_{i 3}\right)\right]^{T}$, and an auxiliary dynamic system is defined as follows:

$$
\begin{aligned}
N_{i j}\left(\chi_{i j}\right) & =\chi_{i j}^{2} \cos \chi_{i j}, \\
\dot{\chi}_{i j} & =\gamma_{\chi i} \bar{\tau}_{c i j} z_{3 i j},
\end{aligned}
$$

where $j=1,2,3$ and design parameter $\gamma_{\chi i}>0$.

The control law is designed as

$$
\begin{aligned}
& \tau_{c i}=T_{i} N_{i} \bar{\tau}_{c i}, \\
& \bar{\tau}_{c i}=-K_{3 i} z_{3 i}+\frac{\xi_{i}}{T_{i}} \tau_{i}+\dot{\alpha}_{i}-z_{2 i},
\end{aligned}
$$

where control gain matrix $K_{3 i}=\operatorname{diag}\left(k_{31 i}, k_{32 i}, k_{33 i}\right)>0$.

Theorem 1. Consider a string of N+1 USVs modeled as (1) and (2) with Assumptions 1 and 2, if the initial condition satisfies $V_{i}(t) \leq B_{0}$ with $B_{0}$ being any positive constant, by adjusting the design parameters $k_{d i}, k_{\psi i}, K_{2 i}, K_{3 i}, \Gamma_{i}, \sigma_{i}$, and $\gamma_{\chi i}$; then, under virtual control law (17) and control law (25) with adaptive updating law (20), the following properties hold:

The output constraint is never violated; i.e., $0<d_{i, \min c o l}<d_{i} \leq d_{i, \max c o m}, \forall t>0$. The constraints on collision avoidance and connectivity maintenance are guaranteed between two consecutive marine vehicles.

The formation errors $e_{d i}$ and $e_{\psi i}$, the tracking error $z_{2 i}$, and weigh error $\tilde{W}_{i}$ converge to a small neighborhood of zero. 
All signals of the MSVs platoon formation closed-loop are bounded.

Remark 1. Vessel train configurations of inland waterways and port areas are an innovative shipping scheme to meet the transportation demand. Although similar works have been developed in robotics and vehicular technology domains, few researchers investigated the waterborne platooning. In the platoon-operating mode, the follower vessels can track the leader in LOS guidance. By employing BLF, collision avoidance is handled to keep a safe distance. Effective communication in predetermined configuration is also taken into account in the design. Besides, input saturation has been dealt with due to the requirement on thrusters.

We present here an analysis based on the obtained platoon controller structure and derive a set of constraints to satisfy platoon objectives. To guarantee the output constraints, it is necessary to ensure that the errors defined in (11) are not violated and all signals are bounded. Therefore, BLF can be utilized in constraints of LOS rang and angle for MSVs to prevent constraint violation. Consider the symmetric barrier Lyapunov function candidate as

$$
V_{1 i}=\frac{1}{2} \ln \frac{k_{a i}^{2}}{k_{a i}^{2}-e_{d i}^{2}}+\frac{1}{2} \ln \frac{k_{b i}^{2}}{k_{b i}^{2}-e_{\psi i}^{2}},
$$

where $k_{a i}$ and $k_{b i}$ are positive constants used to constrain $e_{d i}$ and $e_{\psi i}$, i.e., $\left|e_{d i}\right|<k_{a i}$ and $\left|e_{\psi i}\right|<k_{b i}$, respectively. Differentiating $V_{1 i}$ with respect to time, we have

$$
\dot{V}_{1 i}=\frac{e_{d i} \dot{e}_{d i}}{k_{a i}^{2}-e_{d i}^{2}}+\frac{e_{\psi i} \dot{e}_{\psi i}}{k_{b i}^{2}-e_{\psi i}^{2}} .
$$

Substituting equations (12) and (17) into equation (27) yields

$$
\dot{V}_{1 i}=-k_{d i} e_{d i}^{2}-k_{\psi i} e_{\psi i}^{2}+\Theta_{1 i}
$$

where

$$
\Theta_{1 i}=\frac{e_{d i}\left(-z_{21 i} \cos \left(\psi_{i}-\varphi_{i}\right)+z_{22 i} \sin \left(\psi_{i}-\varphi_{i}\right)\right)}{k_{a i}^{2}-e_{d i}^{2}}+\frac{e_{\psi i}\left(-z_{23 i}\right)}{k_{b i}^{2}-e_{\psi i}^{2}} .
$$

Consider the Lyapunov function candidate $V_{2 i}$ as follows:

$$
V_{2 i}=V_{1 i}+\frac{1}{2} z_{2 i}^{T} M_{i} z_{2 i}+\frac{1}{2} \sum_{i=1}^{3} \tilde{W}_{i}^{T} \Gamma_{i}^{-1} \tilde{W}_{i}
$$

where $\widetilde{W}_{i}=\widehat{W}_{i}-W_{i}^{*}$. Then, the derivation of $V_{2 i}$ is

$$
\dot{V}_{2 i}=-k_{d i} e_{d i}^{2}-k_{\psi i} e_{\psi i}^{2}+z_{2 i}^{T} \Theta_{2}+z_{2 i}^{T}\left[z_{3 i}-C_{i}\left(v_{i}\right) v_{i}-M_{i} \dot{\alpha}_{i}+d_{i}+W_{i}^{* T} H_{i}\left(Z_{i}\right)+\varepsilon_{i}\left(Z_{i}\right)+\alpha_{c i}\right]+\sum_{j=1}^{3} \tilde{W}_{i j}^{T} \Gamma_{i j}^{-1} \dot{\hat{W}}_{i j}
$$

where

$$
\Theta_{2 i}=\left[\frac{-e_{d i} \cos \left(\psi_{i}-\varphi_{i}\right)}{k_{a i}^{2}-e_{d i}^{2}} \frac{e_{d i} \sin \left(\psi_{i}-\varphi_{i}\right)}{k_{a i}^{2}-e_{d i}^{2}} \frac{-e_{\psi i}}{k_{b i}^{2}-e_{\psi i}^{2}}\right]^{T} .
$$

For adaptive law (20), there exists a compact set

$$
\Omega_{w i}=\left\{\widehat{W}_{i} \mid\left\|\widehat{W}_{i}\right\| \leq \frac{s_{i}}{\sigma_{i}}\right\}
$$

where $\left\|H_{i}\left(Z_{i}\right)\right\| \leq s_{i}$ with $s_{i}>0$, such that $\Omega_{w i}, \forall t \geq 0$, provided that $\widehat{W}_{i}(0) \in \Omega_{w i}$.

From Lemma 2, we can know that

$$
\left\|\widetilde{W}_{i}\right\|=\left\|\widehat{W}_{i}-W_{i}^{*}\right\| \leq\left\|\widehat{W}_{i}\right\|+\left\|W_{i}^{*}\right\| \leq \frac{s_{i}}{\sigma_{i}}+\left\|W_{i}^{*}\right\|=ळ_{i}
$$

Substituting control law (25), then according to the Young inequality and Lemma 1, we have

$$
\begin{aligned}
\dot{V}_{2 i} & =-k_{d i} e_{d i}^{2}-k_{\psi i} e_{\psi i}^{2}-\frac{k_{a i}^{2} e_{d i}^{2}}{k_{a i}^{2}-e_{d i}^{2}}-\frac{k_{b i}^{2} e_{\psi i}^{2}}{k_{b i}^{2}-e_{\psi i}^{2}}-z_{2 i}^{T} K_{2 i} z_{2 i}-z_{2 i}^{T} \varepsilon_{i}\left(Z_{i}\right)+z_{2 i}^{T} z_{3 i}+z_{2 i}^{T} d_{i}+\sum_{i=1}^{3} \tilde{W}_{i}^{T}\left(H_{i}\left(Z_{i}\right) z_{2 i}-\sigma_{i}\left|z_{2 i}\right| \widehat{W}_{i}\right) \\
& \leq-k_{d i} \ln \frac{k_{a i}^{2}}{k_{a i}^{2}-e_{d i}^{2}}-k_{\psi i} \ln \frac{k_{b i}^{2}}{k_{b i}^{2}-e_{\psi i}^{2}}+z_{2 i}^{T} d_{i}+z_{2 i}^{T} z_{3 i}-z_{2 i}^{T}\left(K_{2 i}-I\right) z_{2 i}+\frac{1}{2}\left\|\varepsilon_{i}\left(Z_{i}\right)\right\|^{2}+\sum_{i=1}^{3} \frac{\sigma_{i}^{2}}{8}\left(\left\|W_{i}^{*}\right\|^{4}+\frac{\sigma_{i}^{2}}{8} \omega_{i}^{4}-2\left\|W_{i}^{*}\right\|^{2}\left\|\widetilde{W}_{i}\right\|^{2}\right) .
\end{aligned}
$$


Consider the following Lyapunov function candidate as follows:

$$
V_{3 i}=\frac{1}{2} z_{3 i}^{T} z_{3 i}
$$

Taking the time derivative of $V_{3 i}$ yields

$$
\begin{aligned}
\dot{V}_{3 i} & =z_{3 i}^{T}\left[\xi_{i}\left(-\frac{1}{T_{i}} \tau_{i}+\frac{1}{T_{i}} \tau_{c i}\right)-\dot{\alpha}_{i}\right] \\
& =-z_{3 i}^{T} K_{3 i} z_{3 i}-z_{3 i}^{T} z_{2 i}+z_{3 i}^{T}\left(\frac{\xi_{i}}{T_{i}} \tau_{c i}-\bar{\tau}_{c i}\right) \\
& =-z_{3 i}^{T} K_{3 i} z_{3 i}-z_{3 i}^{T} z_{2 i}+\frac{1}{\gamma_{\chi i}} \sum_{j=1}^{3}\left(\xi_{i j} N_{i j}-1\right) \dot{\chi}_{i j} .
\end{aligned}
$$

The overall Lyapunov function is

$$
V_{i}=V_{2 i}+V_{3 i}
$$

So we can obtain

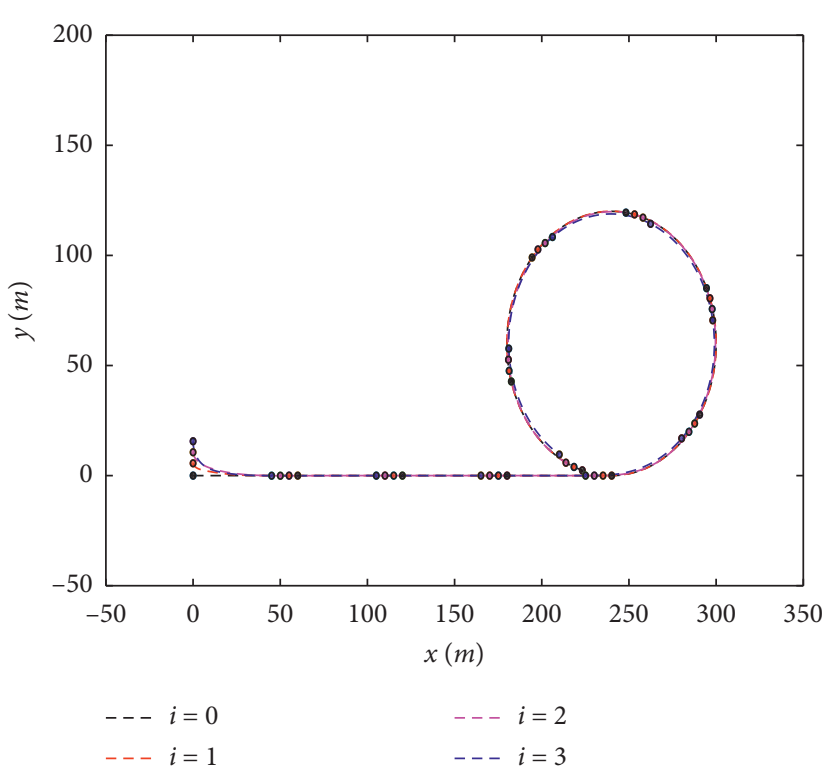

Figure 3: Trajectories of the MSVs.

$$
\begin{aligned}
\dot{V}_{i} \leq & -k_{d i} \ln \frac{k_{a i}^{2}}{k_{a i}^{2}-e_{d i}^{2}}-k_{\psi i} \ln \frac{k_{b i}^{2}}{k_{b i}^{2}-e_{\psi i}^{2}}-z_{3 i}^{T} K_{3 i} z_{3 i}-z_{2 i}^{T}\left(K_{2 i}-\frac{3}{2} I\right) z_{2 i}+\frac{1}{2}\left\|\varepsilon_{i}\left(Z_{i}\right)\right\|^{2} \\
& +\sum_{i=1}^{3} \frac{\sigma_{i}^{2}}{8}\left(\left\|W_{i}^{*}\right\|^{4}+\frac{\sigma_{i}^{2}}{8} \oplus_{i}^{4}-2\left\|W_{i}^{*}\right\|^{2}\left\|\widetilde{W}_{i}\right\|^{2}\right)+\frac{1}{\gamma_{\chi i}} \sum_{j=1}^{3}\left(\xi_{i j} N_{i j}-1\right) \dot{\chi}_{i j}+\frac{1}{2} D_{i \max } \leq-\rho_{i} V_{i}+C_{i}+\frac{1}{\gamma_{\chi i}} \sum_{j=1}^{3}\left(\xi_{i j} N_{i j}-1\right) \dot{\chi}_{i j},
\end{aligned}
$$

where

$$
\rho_{i}=\min \left\{2 k_{d i}, 2 k_{\psi i}, \frac{2 \lambda_{\min }\left(K_{2 i}\right)-3}{\lambda_{\max }\left(M_{i}\right)}, 2 \lambda_{\min }\left(K_{3 i}\right), \frac{\sigma_{i}^{2}\left\|W_{i}^{*}\right\|^{2}}{2 \lambda_{\max }\left(\Gamma_{i}^{-1}\right)}\right\},
$$

$$
C_{i}=\frac{1}{2}\left\|\bar{\varepsilon}_{i}\right\|^{2}+\frac{1}{2} D_{i \max }+\sum_{i=1}^{3}\left(\frac{\sigma_{i}^{2}}{8}\left\|W_{i}^{*}\right\|^{4}+\frac{\sigma_{i}^{2}}{8}{\aleph_{i}^{4}}^{4}\right)
$$

where the minimum and maximum eigenvalues of matrix $\star$ are denoted by $\lambda_{\min }(\star)$ and $\lambda_{\max }(\star)$, respectively.

$$
0 \leq V_{i}(t) \leq \frac{C_{i}}{\rho_{i}}+\left[V_{i}(0)-\frac{C_{i}}{\rho_{i}}\right] e^{-\rho_{i} t}+\frac{e^{-\rho_{i} t}}{\gamma_{\chi i}} \int_{0}^{t} \sum_{j=1}^{3}\left(\xi_{i j} N_{i j}-1\right) \dot{\chi}_{i j} e^{-\rho_{i} s} \mathrm{~d} s .
$$

From (42) and Lemma 2, it is obvious that $V(t)$ and $\chi_{i}$ are uniformly ultimately bounded for all $V(0) \leq B_{0}$ on $\left[0, t_{f}\right)$. Therefore, we can deduce that the closed-loop errors $e_{d i}, e_{\psi i}, z_{2 i}$, and $\widetilde{W}_{i}$ are also uniformly ultimately bounded.

\section{Simulation Examples}

In this section, a string of $4 \mathrm{MSV}$ s is used for the numerical simulations to demonstrate the effectiveness of the proposed method. The communication relationship of the 4 MSVs is shown in Figure 3. The 3-DOF rotation matrix $J_{i}$ is defined as

$$
J_{i}\left(\psi_{i}\right)=\left[\begin{array}{ccc}
\cos \left(\psi_{i}\right) & -\sin \left(\psi_{i}\right) & 0 \\
\sin \left(\psi_{i}\right) & \cos \left(\psi_{i}\right) & 0 \\
0 & 0 & 1
\end{array}\right] .
$$

$M_{i} \in \mathbb{R}^{3 \times 3}$ is a symmetric positive definite inertia matrix specified as

$$
M_{i}=\left[\begin{array}{ccc}
m_{11 i} & 0 & 0 \\
0 & m_{22 i} & m_{23 i} \\
0 & m_{32 i} & m_{33 i}
\end{array}\right]
$$

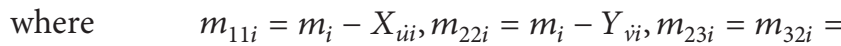
$m_{i} x_{g i}-Y_{r i}, m_{33 i}=I_{z i}-N_{r i}$, and the mass of the $i$-th marine vehicle is $m_{i}$, and the $i$ - th marine vehicle's inertia matrix in the body-fixed frame is $I_{z i} . D_{i}\left(v_{i}\right)$ is a hydrodynamic damping matrix specified as

$$
D_{i}\left(v_{i}\right)=\left[\begin{array}{ccc}
d_{11 i} & 0 & 0 \\
0 & d_{22 i} & d_{23 i} \\
0 & d_{32 i} & d_{33 i}
\end{array}\right] \text {, }
$$

where 


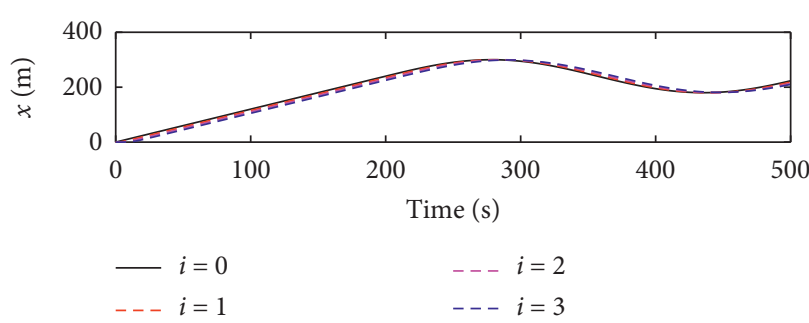

(a)

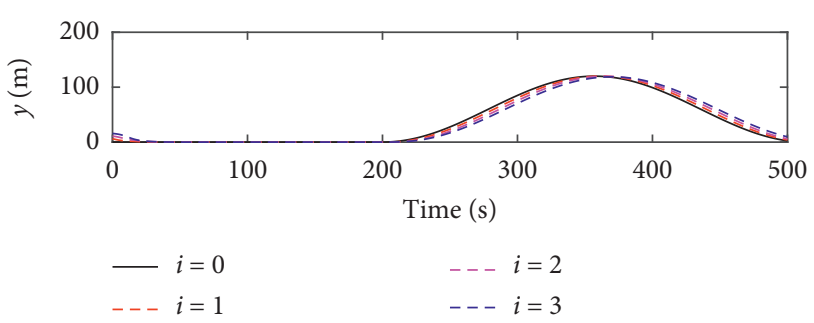

(b)

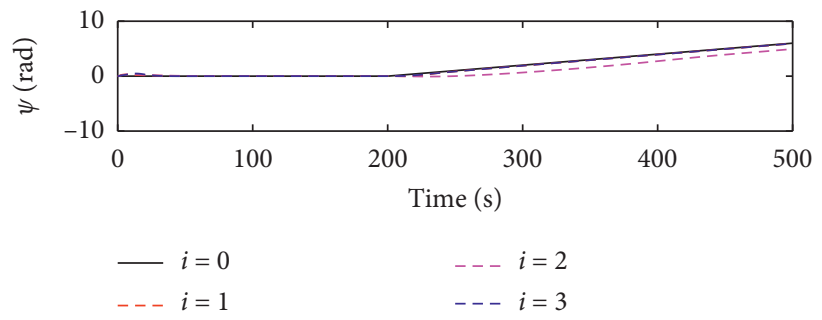

(c)

Figure 4: Positions and orientations of the MSVs.

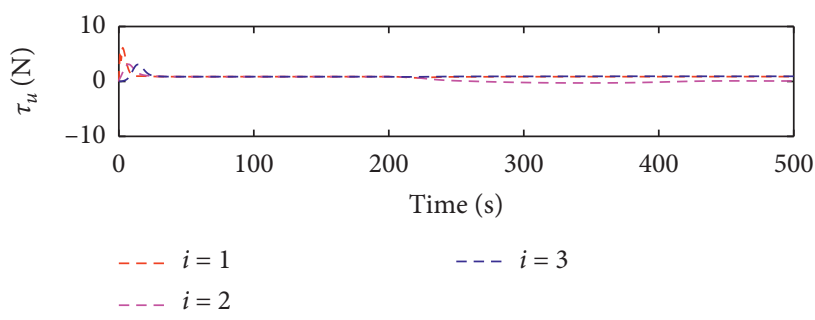

(a)

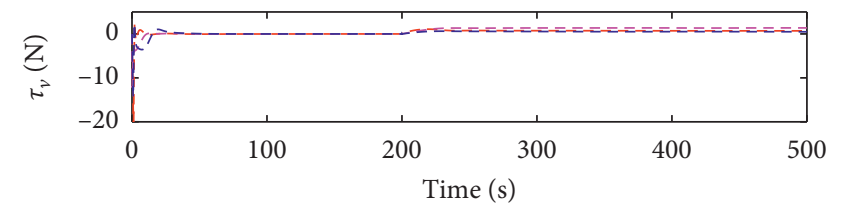

$---i=1 \quad---i=3$

(b)

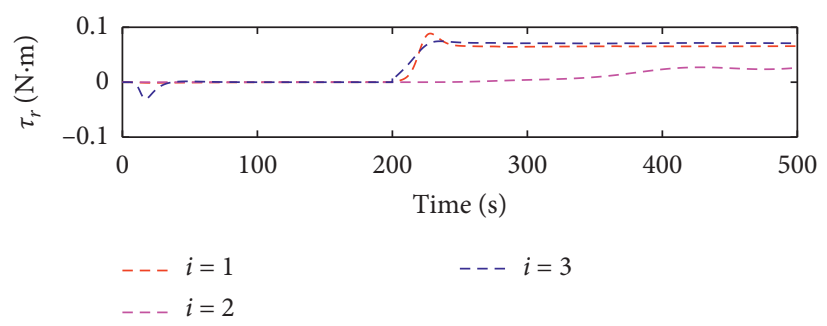

(c)

Figure 5: Control inputs.

$$
\begin{aligned}
d_{11 i}\left(v_{i}\right) & =-\left(X_{u_{i}}+X_{\left|u_{i}\right| u_{i} \mid}\left|u_{i}\right|+X_{u_{i} u_{i} u_{i}} u_{i}^{2}\right), \\
d_{22 i}\left(v_{i}, r_{i}\right) & =-\left(Y_{v_{i}}+Y_{\left|v_{i}\right| v_{i} \mid}\left|v_{i}\right|+Y_{|r i| v_{i}}\left|r_{i}\right|\right), \\
d_{23 i}\left(v_{i}, r_{i}\right) & =-\left(Y_{r_{i}}+Y_{\left|v_{i}\right| r_{i} \mid}\left|v_{i}\right|+Y_{\left|r_{i}\right| r_{i} \mid}\left|r_{i}\right|\right), \\
d_{32 i}\left(v_{i}, r_{i}\right) & =-\left(N_{v_{i}}+N_{\left|v_{i}\right| v_{i} \mid}\left|v_{i}\right|+N_{\left|r_{i}\right| v_{i} \mid}\left|r_{i}\right|\right), \\
d_{33 i}\left(v_{i}, r_{i}\right) & =-\left(N_{r_{i}}+N_{\left|v_{i}\right| r_{i} \mid}\left|v_{i}\right|+N_{\left|r_{i}\right| r_{i} \mid}\left|r_{i}\right|\right) .
\end{aligned}
$$

The matrix $C_{i}\left(v_{i}\right)$ is specified as

$$
C_{i}\left(v_{i}\right)=\left[\begin{array}{ccc}
0 & 0 & -m_{22 i} v_{i}-m_{23 i} r_{i} \\
0 & 0 & m_{11 i} u_{i} \\
m_{22 i} v+m_{23 i} r_{i} & -m_{11 i} u_{i} & 0
\end{array}\right] .
$$

The marine vehicle model parameters in this simulation are exploited from Cybership-II [37].

The initial states of the MSVs are given as $\eta_{0}=[0,0,0]^{T}$, $\eta_{1}=[0,5.6,0]^{T}, \eta_{2}=[0,10.6,0]^{T}, \eta_{3}=[0,15.6,0]^{T}$, and $v_{1}=v_{2}=v_{3}=0$. From Figure 3, the MSVs formate in platoon during the moving process. The desired positions and the orientations of MSVs are shown in Figure 4. Figure 5 shows the control inputs, which satisfy the design 


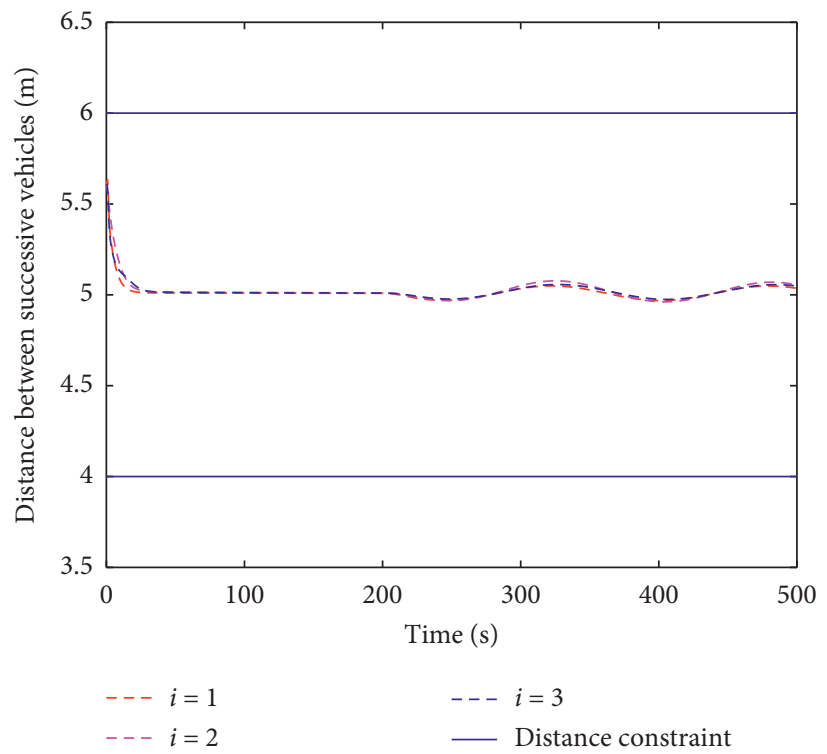

Figure 6: The distances between successive vehicles and constraints.

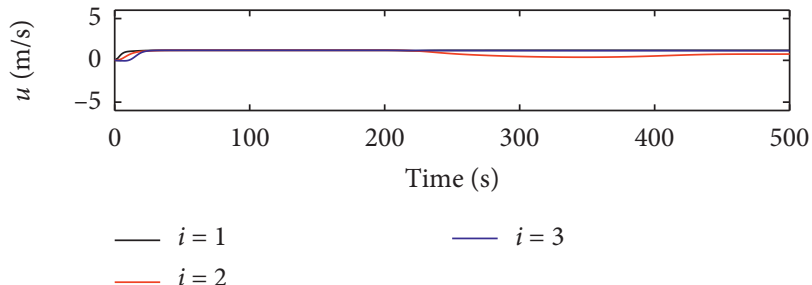

(a)

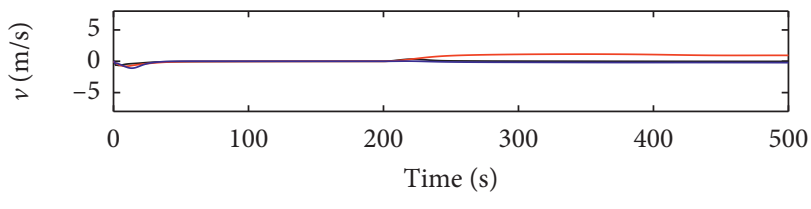

$-i=1$

$-i=2$

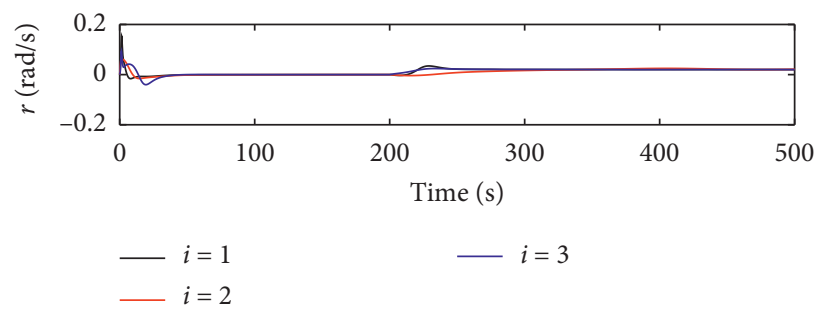

(c)

Figure 7: Velocities of the MSVs.

requirement. The distances between the follower and the leader are shown in Figure 6. The outputs are constrained between the boundaries effectively. The desired distance between the successive vehicles is $5 \mathrm{~m}$. The minimum collision distance is set as $4 \mathrm{~m}$, and the maximum connectivity distance is set as $6 \mathrm{~m}$. Figure 7 shows the velocities information among MSVs. Simulation results demonstrate the efficacy of the proposed method.

\section{Conclusions}

In this paper, decentralized formation control with input and output constraints have been proposed for a string MSVs. In order to achieve collision avoidance and connectivity maintenance, LOS range and angle errors were presented in the controller design. A BLF has been employed to prevent constraint violation. Next, to deal with the model uncertainties, the adaptive neural networks are applied in the backstepping control scheme due to their superior approximation capability. Furthermore, the nonlinear term arising from the input saturation is compensated by using a Nussbaum function. The analysis of uniformly ultimate boundedness for the closed-loop platoon system was provided by choosing the design parameters. Finally, the proposed algorithm was applied to MSVs to illustrate the feasibility of the theoretical results.

\section{Data Availability}

The simulation data used to support the findings of this study are included within the article. 


\section{Conflicts of Interest}

The authors declare that they have no conflicts of interest.

\section{Acknowledgments}

This research was funded by the National Natural Science Foundation of China under Grant 51709027 and the Chinese Scholarship Council of the Ministry of Education.

\section{References}

[1] K.-K. Oh, M.-C. Park, and H.-S. Ahn, "A survey of multiagent formation control," Automatica, vol. 53, pp. 424-440, 2015.

[2] Z. Zuo, "Nonsingular fixed-time consensus tracking for second-order multi-agent networks," Automatica, vol. 54, pp. 305-309, 2015.

[3] D. Li, S. S. Ge, W. He, G. Ma, and L. Xie, "Multilayer formation control of multi-agent systems," Automatica, vol. 109, p. 108558, 2019.

[4] J.-W. Kwon and D. Chwa, "Adaptive bidirectional platoon control using a coupled sliding mode control method," IEEE Transactions on Intelligent Transportation Systems, vol. 15, no. 5, pp. 2040-2048, 2014.

[5] M. Defoort, T. Floquet, A. Kokosy, and W. Perruquetti, "Sliding-mode formation control for cooperative autonomous mobile robots," IEEE Transactions on Industrial Electronics, vol. 55, no. 11, pp. 3944-3953, 2008.

[6] Y. Cao, W. Ren, and Z. Meng, "Decentralized finite-time sliding mode estimators and their applications in decentralized finite-time formation tracking," Systems \& Control Letters, vol. 59, no. 9, pp. 522-529, 2010.

[7] D. Li, G. Ma, W. He, W. Zhang, C. Li, and S. S. Ge, "Distributed coordinated tracking control of multiple EulerLagrange systems by state and output feedback," IET Control Theory \& Applications, vol. 11, no. 14, pp. 2213-2221, 2017.

[8] Q. Zhang and H. H. T. Liu, "UDE-based robust command filtered backstepping control for close formation flight," IEEE Transactions on Industrial Electronics, vol. 65, no. 11, pp. 8818-8827, 2018.

[9] Y.-Y. Chen and Y.-P. Tian, "A backstepping design for directed formation control of three-coleader agents in the plane," International Journal of Robust and Nonlinear Control, vol. 19, no. 7, pp. 729-745, 2009.

[10] H. G. Tanner, G. J. Pappas, and V. Kumar, "Leader-to-Formation stability," IEEE Transactions on Robotics and Automation, vol. 20, no. 3, pp. 443-455, 2004.

[11] J. R. T. Lawton, R. W. Beard, and B. J. Young, "A decentralized approach to formation maneuvers," IEEE Transactions on Robotics and Automation, vol. 19, no. 6, pp. 933-941, 2003.

[12] D. Jia, K. Lu, J. Wang et al., "A survey on platoon-based vehicular cyber-physical systems[J]," IEEE Communications Surveys \& Tutorials, vol. 18, no. 1, pp. 263-284, 2015.

[13] S.-L. Dai, S. He, H. Lin, and C. Wang, "Platoon Formation control with prescribed performance guarantees for USVs," IEEE Transactions on Industrial Electronics, vol. 65, no. 5, pp. 4237-4246, 2018.

[14] S. E. Li, Y. Zheng, K. Li et al., "An overview of vehicular platoon control under the four-component framework," in Proceedings of the 2015 IEEE Intelligent Vehicles Symposium (IV), pp. 286-291, IEEE, Seoul, South Korea, July 2015.

[15] Y. Zhou, S. Ahn, M. Wang et al., "Stabilizing mixed vehicular platoons with connected automated vehicles: an h-infinity approach," Transportation Research Part B: Methodological, vol. 132, 2019.

[16] R. Florin and S. Olariu, "A survey of vehicular communications for traffic signal optimization," Vehicular Communications, vol. 2, no. 2, pp. 70-79, 2015.

[17] M. Karimi, H. Mokhtari, and M. R. Iravani, "Wavelet based on-line disturbance detection for power quality applications," IEEE Transactions on Power Delivery, vol. 15, no. 4, pp. 1212-1220, 2000.

[18] C. Ning and F. You, "Data-driven adaptive robust unit commitment under wind power uncertainty: a bayesian nonparametric approach," IEEE Transactions on Power Systems, vol. 34, no. 3, pp. 2409-2418, 2019.

[19] C. Zhong, Z. Chen, and Y. Guo, "Attitude control for flexible spacecraft with disturbance rejection," IEEE Transactions on Aerospace and Electronic Systems, vol. 53, no. 1, pp. 101-110, 2017.

[20] L. W. Cook and J. P. Jarrett, "Optimization using multiple dominance criteria for aerospace design under uncertainty," AIAA Journal, vol. 56, no. 12, pp. 4965-4976, 2018.

[21] S. Liu, Y. Liu, X. Liang et al., "Uncertainty observation-based adaptive succinct fuzzy-neuro dynamic surface control for trajectory tracking of fully actuated underwater vehicle system with input saturation," Nonlinear Dynamics, vol. 98, no. 3, pp. 1-17, 2019.

[22] S. Skjong and E. Pedersen, "Model-based control designs for offshore hydraulic winch systems," Ocean Engineering, vol. 121, pp. 224-238, 2016.

[23] S. Ghapani, J. Mei, W. Ren, Y. Song et al., "Fully distributed flocking with a moving leader for Lagrange networks with parametric uncertainties," Automatica, vol. 67, pp. 67-76, 2016.

[24] A. R. Mehrabian and K. Khorasani, "Constrained distributed cooperative synchronization and reconfigurable control of heterogeneous networked Euler-Lagrange multi-agent systems," Information Sciences, vol. 370-371, pp. 578-597, 2016.

[25] X. Ge, Q. L. Han, and X. M. Zhang, "Achieving cluster formation of multi-agent systems under aperiodic sampling and communication delays," IEEE Transactions on Industrial Electronics, vol. 65, no. 4, pp. 3417-3426, 2017.

[26] B. L. Zhang, Q. L. Han, X. M. Zhang et al., "Sliding mode control with mixed current and delayed states for offshore steel jacket platforms," IEEE Transactions on Control Systems Technology, vol. 22, no. 5, pp. 1769-1783, 2013.

[27] R. Ji, J. Ma, D. Li et al., "Finite-time adaptive output feedback control for MIMO nonlinear systems with actuator faults and saturations," IEEE Transactions on Fuzzy Systems, 2020.

[28] X. Liang, M. Hou, and G. Duan, "Adaptive dynamic surface control for integrated missile guidance and autopilot in the presence of input saturation," Journal of Aerospace Engineering, vol. 28, no. 5, Article ID 04014121, 2014.

[29] J. Ghommam, S. E. Ferik, and M. Saad, "Robust adaptive pathfollowing control of underactuated marine vessel with offtrack error constraint," International Journal of Systems Science, vol. 49, no. 7, pp. 1540-1558, 2018.

[30] Z. Yin, W. He, and C. Yang, "Tracking control of a marine surface vessel with full-state constraints," International Journal of Systems Science, vol. 48, no. 3, pp. 535-546, 2017.

[31] Z. Zhao, W. He, and S. S. Ge, "Adaptive neural network control of a fully actuated marine surface vessel with multiple output constraints," IEEE Transactions on Control Systems Technology, vol. 22, no. 4, pp. 1536-1543, 2014. 
[32] M. Chen, S. S. Ge, and B. Ren, "Adaptive tracking control of uncertain MIMO nonlinear systems with input constraints," Automatica, vol. 47, no. 3, pp. 452-465, 2011.

[33] C. Wen, J. Zhou, Z. Liu, and H. Su, "Robust adaptive control of uncertain nonlinear systems in the presence of input saturation and external disturbance," IEEE Transactions on Automatic Control, vol. 56, no. 7, pp. 1672-1678, 2011.

[34] T. Li, R. Li, and J. Li, "Decentralized adaptive neural control of nonlinear interconnected large-scale systems with unknown time delays and input saturation," Neurocomputing, vol. 74, no. 14-15, pp. 2277-2283, 2011.

[35] H. Wang, D. Wang, and Z. Peng, "Adaptive dynamic surface control for cooperative path following of marine surface vehicles with input saturation," Nonlinear Dynamics, vol. 77, no. 1-2, pp. 107-117, 2014.

[36] Z. Zheng, Y. Huang, L. Xie et al., "Adaptive trajectory tracking control of a fully actuated surface vessel with asymmetrically constrained input and output," IEEE Transactions on Control Systems Technology, vol. 26, no. 5, pp. 1851-1859, 2018.

[37] K. P. Tee and S. S. Ge, "Control of fully actuated ocean surface vessels using a class of feedforward approximators," IEEE Transactions on Control Systems Technology, vol. 14, no. 4, pp. 750-756, 2006. 